\title{
11 Comparing the Growth Dynamics of Real and Virtual Cities
}

\author{
Narushige Shiode and Paul Torrens
}

\section{CONTENTS}

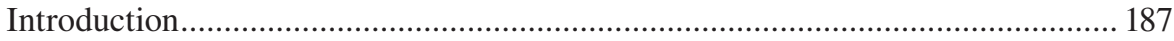

A Tale of Two Cities-Alphaworld and Austin, Texas .......................................... 189

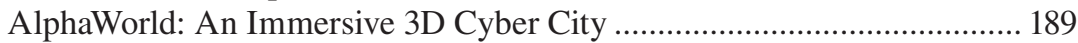

Austin, Texas: A Fast-Growing Real City ............................................... 192

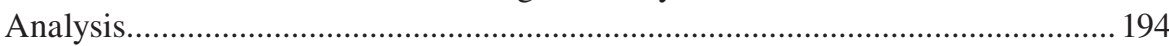

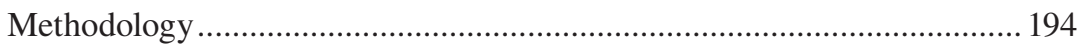

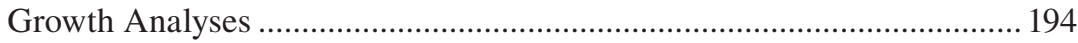

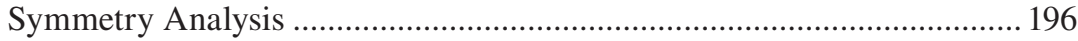

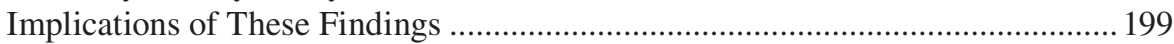

Location, Location, Location! ….................................................................. 199

Building the Impossible ...................................................................... 199

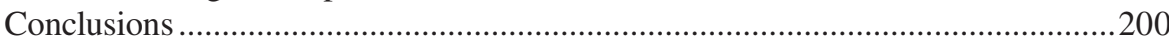

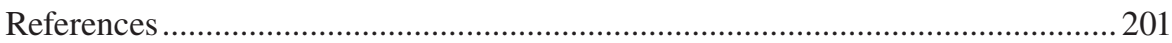

\section{INTRODUCTION}

Virtual cities were first conceptualized in science fiction literature. They were popularized by cyberpunk authors such as Neal Stephenson and William Gibson and their tales of vast "metaverses" and "cyberspaces," composed of bits rather than atoms, sprawling like megalopolises (Stephenson 1993), towering with information skyscrapers (Gibson 1984), with firewalls crafted to resemble medieval cities (Gibson 1996). As the stories go, these cyber cities are inhabited by real people, rendered in virtual space as digital avatars of various descriptions while maintaining a corporeal presence in the real, tangible world.

In recent years, the boundary between fact and science fiction has dissolved to some extent. Several such virtual cities have been constructed and continue to evolve online (Activeworlds 2006). The popularity of virtual cities is set to grow still further, catalyzed by the present enthusiasm for Massively Multiplayer Online Role-Playing 
Games (MMORPG) and, more generally, for social network services (SNS). Several high-profile virtual cities have been opened, or are imminently forthcoming (Linden Lab 2007; There 2007). In addition, the advent of online 3D city models triggered by the development of various Web-mapping services has increased the opportunity for use of virtual cities for virtual tourism, way-finding, data sharing, and online participatory and decision-making purposes for a variety of applications (Shiode 2001; Shiode and Yin 2007).

Virtual cities have obvious appeal in the context of gaming and entertainment, but they also have other uses that are tied to planning, marketing, designing, promoting, predicting, and assessing and evaluating the various factors surrounding an urban environment and, thereby, forecasting a possible future form of the city (Batty et al. 2000). In particular, researchers in urban studies have long used simulation as a planning support tool (Torrens 2002). The rationale is that simulations can function as virtual laboratories for testing hypotheses, ideas, plans, and policies in artificial urban systems, in ways that are not feasible or may be impractical in the real world. Simulations of this nature generally portray urban systems in very abstract terms — although that is beginning to change; see Benenson and Torrens (2004) for more details on this discussion. In particular, the representation of urban spaces, structures, patterns, and morphologies in traditional urban simulation contexts is often cursory.

Cyber cities that are developed in the MMORPG tradition offer fantastic potential as planning-support tools, because they are populated by real people, replacing the synthetic, "mean individuals" (Wrigley et al. 1996) commonly found in urbansimulation models. The emphasis in classic urban simulation is on mimicking urban processes, generally using a variety of algorithms, equations, or transition rules. In virtual cities, by contrast, urban spaces are configured and generated by real people. Citizens of a virtual city are often collective individual users accessing the virtual environment through their own computer consoles. These virtual citizens, also referred to as netizens, act as designers, architects, planners, developers, policy-makers, and inhabitants in their virtual urban environment (Shiode 2001). Virtual cities thus open up a new world of research potential. Yet little work has been conducted in this area. Research into social interactions, discourse, and behavior in online worlds abounds, and is carried out in fields external to urban studies. Nevertheless, little attention has been paid to virtual cities as urban spaces, despite the obvious advantages of using virtual cities as laboratories for research in urban studies (Shiode 2000). The dynamics of urban space represented by the growth trajectory of virtual cities, in particular, would make a perfect ground for studying the growth and life cycle of an urban environment.

This chapter examines the production of a virtual city - the manner in which urban space is developed and populated — in the longest-running virtual city, AlphaWorld. We are particularly interested in determining the extent to which virtual cities in AlphaWorld resemble their real-world counterparts; in this case, Austin, Texas. We chose Austin, as we identified many common features between the two cities. These include the relatively flat topography, comparable scale of their geographical extent, and rapid growth of the urbanized area over recent years. While the insights obtained through this study may not provide sufficient evidence for the general comparison of both groups of cities, virtual and real, they will help us better 
understand the prospect of measuring the patterns of urban development in both environments.

Rather than conducting this study from a narrative standpoint, we have applied more rigorous empirical methodologies - from spatial analysis, complexity studies, and GIScience - and we use these to compare and contrast the virtual and the tangible urban environments. Specifically, we employ techniques from a toolkit that we are applying elsewhere to the evaluation of patterns of suburban sprawl in North American cities (Torrens and Alberti 2000) and regularities in the rank-size scaling of metropolitan areas (Batty and Shiode 2003).

The rest of the chapter will be as follows. Section 2 reports recent trends in the growth of two case cities, AlphaWorld and Austin, Texas. This is followed by analyses and their results presented in Section 3 with an emphasis on the comparison of the relative complexity and symmetry between the two cities. Section 4 brings the paper to a close, discussing the findings, their implications in the context of urban studies, and outlying potential avenues for further exploration.

\section{A TALE OF TWO CITIES-ALPHAWORLD AND AUSTIN, TEXAS}

\section{AlphaWorld: An Immersive 3D Cyber City}

AlphaWorld is an immersive, multi-user, three-dimensional cyber city. Its universe is generated electronically and rendered within the virtual environment of the Internet. Users interface and interact with and within the world by logging in from a networked computer. The world has a surface that extends to an area equivalent to 429,025 sq. $\mathrm{km}$, which is comparable in size to the state of California. AlphaWorld is rendered, in two-dimensional space, as a plane; and its areal extent spreads from a central point, Ground Zero ( $0 \mathrm{~N} \mathrm{OW}$ ) to four corners - from $(32,750 \mathrm{~S} 32,750 \mathrm{E})$ to $(32,750 \mathrm{~N} 32,750 \mathrm{~W})$. Within this plane, users have constructed three-dimensional cities. The world has been active since 1995. It is still thinly populated, but has a rapid growth rate. As of August 8, 2002, there are 127.6 million objects in AlphaWorld, but only 65.1 million unit cells within its boundary (1.52 percent of the total number of cells) contain manmade objects (Roelofs and van der Meulen 2002).

AlphaWorld is a pseudo-three-dimensional environment in that users can "walk about" in the streets and "talk" to other people. The world is incredibly flexible; its users are free to construct their own buildings and interact with other users (detailed information can be found at www.activeworlds.com). Nevertheless, it also retains certain unique spatial features that are not part of the real world, for example, users can enter arbitrary coordinates and instantly "jump" (or teleport) to the desired destination.

Figure 11.1 illustrates changes in the land cover for AlphaWorld over a five-year period. The maps shown in Figure 11.1 cover the area between (1000N 1000W) and (1000S 1000E) of AlphaWorld, an area equivalent to 400 sq. km (roughly 0.3 percent of the total area of AlphaWorld). Ground Zero is at the center of the picture. Considering the maps in a purely visual sense, a number of striking phenomena are apparent. First, the area has obviously undergone dynamic development. This has been both rapid and complex in its manifestation. In particular, the maps illustrate incremental development in a monotonic and consistent fashion, characteristic of 


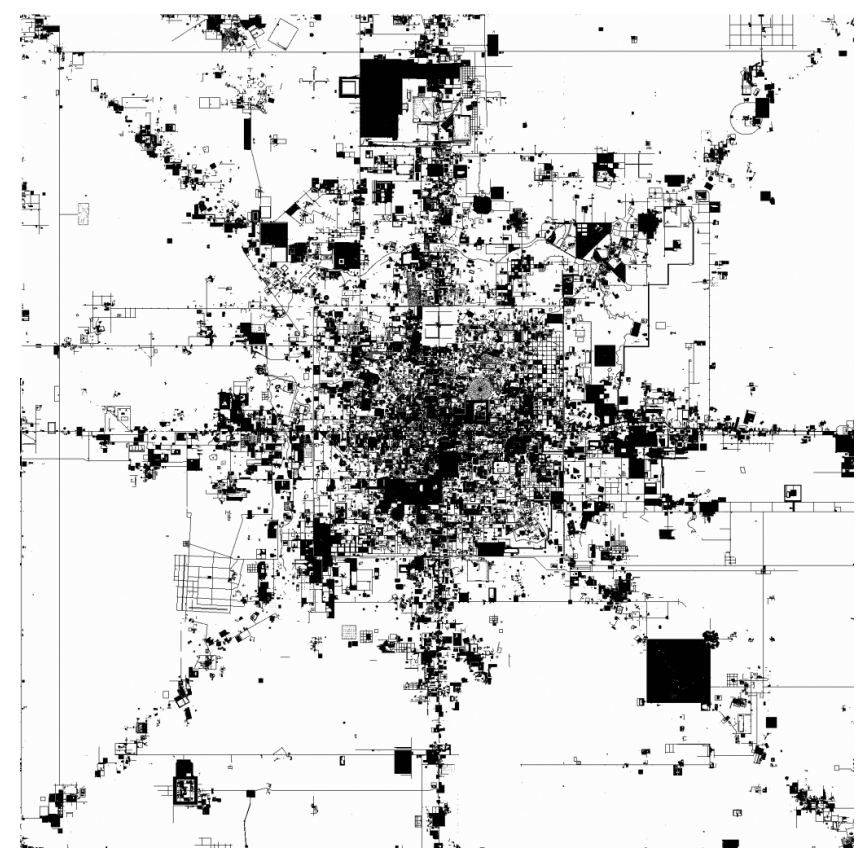

FIGURE 11.1a Building footprints in the central $400 \mathrm{~km}^{2}$ area of AlphaWorld (a) December 1996, (b) February 1998, (c) August 1999, (d) August 2001 (images adapted through a smoothing and reclassification procedure using original images at www.activeworlds.com/ community/maps.asp).

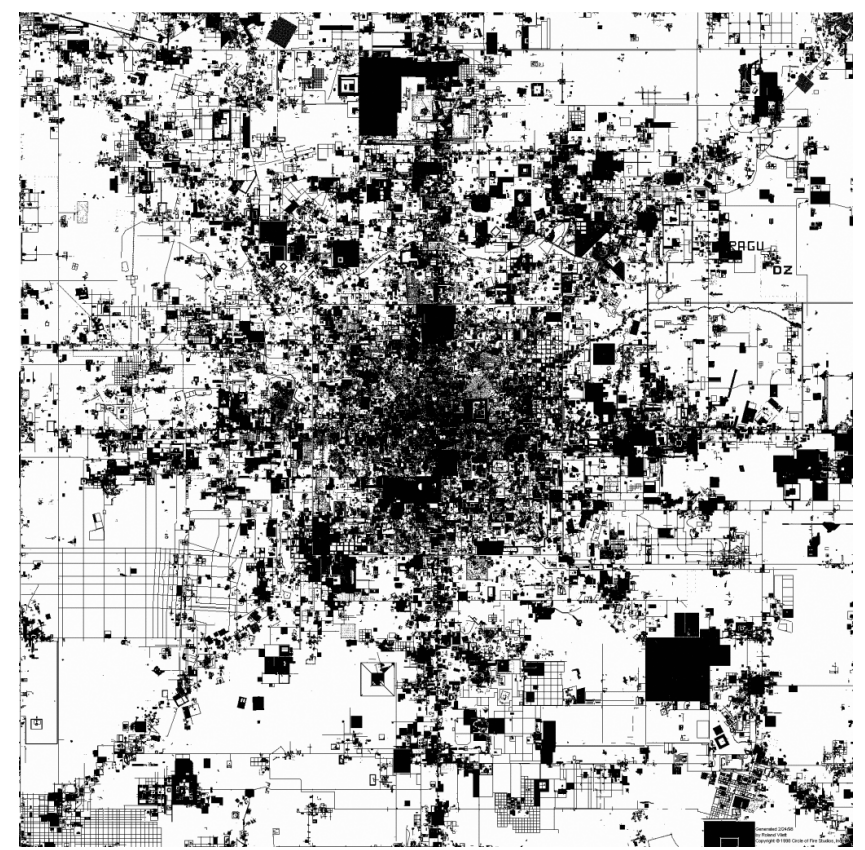

FIGURE 11.1b (Continued). 


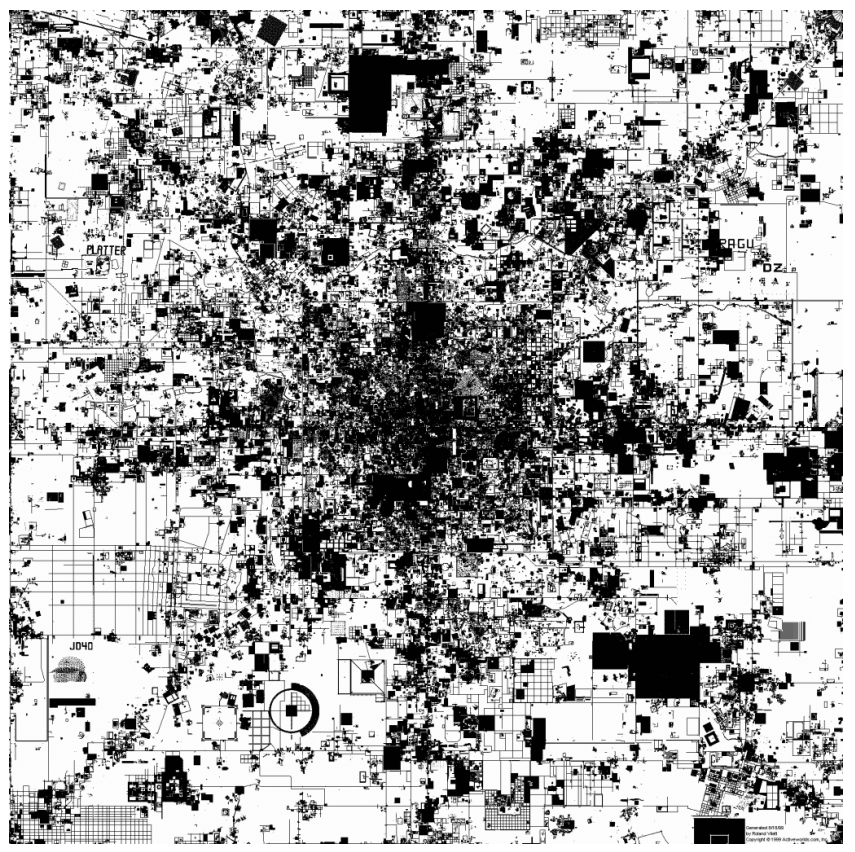

FIGURE 11.1c (Continued).

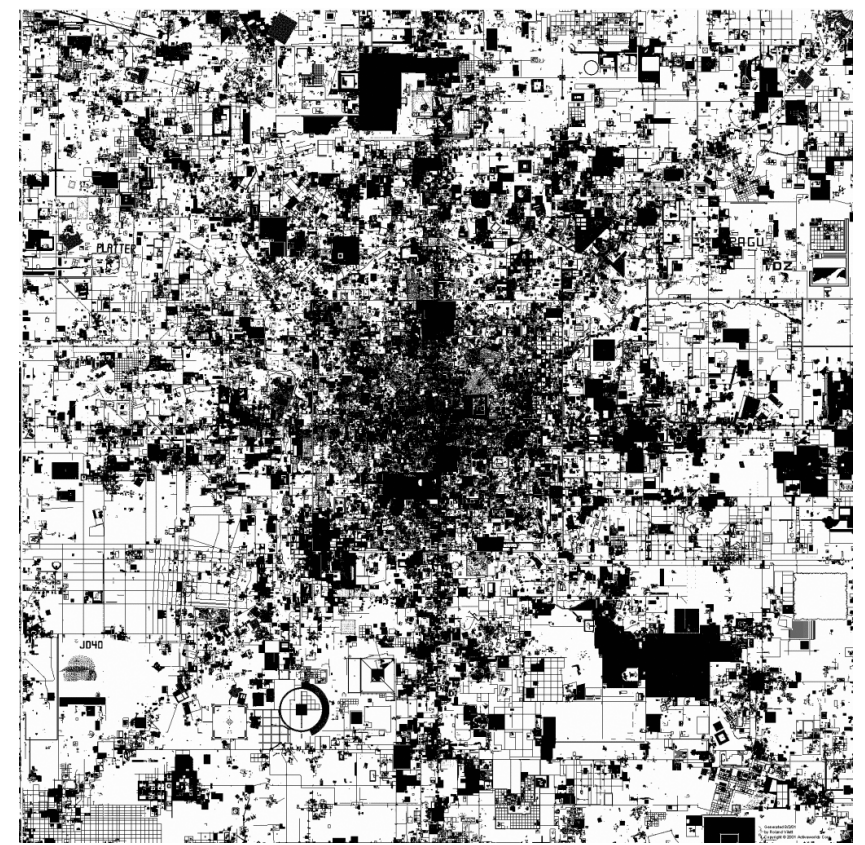

FIGURE 11.1d (Continued). 


\section{TABLE 11.1}

The Top Ten Fastest-Growing Cities in the United States (Source: U.S. Census Bureau, Census 2000 Redistricting Data [P.L. 94-171] Summary File and 1990 Census)

\begin{tabular}{|c|c|c|c|c|c|}
\hline \multirow[b]{2}{*}{ Rank } & \multirow[b]{2}{*}{ Metropolitan Area Name } & \multicolumn{2}{|c|}{ Census Population } & \multicolumn{2}{|c|}{ Change 1990-2000 } \\
\hline & & April 1990 & April 2000 & Number & $\%$ \\
\hline 1 & Las Vegas, NV-AZ & 852,737 & $1,563,282$ & 710,545 & $83.3 \%$ \\
\hline 2 & Naples, FL & 152,099 & 251,377 & 99,278 & $65.3 \%$ \\
\hline 3 & Yuma, AZ & 106,895 & 160,026 & 53,131 & $49.7 \%$ \\
\hline 4 & McAllen—Edinburg_-Mission, TX & 383,545 & 569,463 & 185,918 & $48.5 \%$ \\
\hline 5 & Austin-San Marcos, TX & 846,227 & $1,249,763$ & 403,536 & $47.7 \%$ \\
\hline 6 & Fayetteville—Springdale—Rogers, AR & 210,908 & 311,121 & 100,213 & $47.5 \%$ \\
\hline 7 & Boise City, ID & 295,851 & 432,345 & 136,494 & $46.1 \%$ \\
\hline 8 & Phoenix-Mesa, AZ & $2,238,480$ & $3,251,876$ & $1,013,396$ & $45.3 \%$ \\
\hline 9 & Laredo, TX & 133,239 & 193,117 & 59,878 & $44.9 \%$ \\
\hline 10 & Provo-Orem, UT & 263,590 & 368,536 & 104,946 & $39.8 \%$ \\
\hline
\end{tabular}

classic monocentric cities. Second, development is focused around a central core area, with a high degree of concentration along two central axes and the diagonals. This "starfish" morphology is due to the unique coordinate teleporting system in AlphaWorld - people tend to focus their building activity along the North-South axis and the "equator," and they also tend to build along coordinates with matching numbers (e.g., 123S 123W). Although these regularities are not at all uncommon in real cities, the degree of concentration is staggering in the case of AlphaWorld. We will revisit this issue later through the comparison of frequency spectra and show that AlphaWorld exhibits by far the more symmetric and regular pattern in its urban growth when compared to Austin.

\section{Austin, Texas: A Fast-Growing Real City}

The city of Austin is only the 38th largest city in the United States, occupying 625 sq. $\mathrm{km}$ of land in Southern Texas. Nevertheless, it ranks as the fifth-fastest-growing city in the United States (Table 11.1); its population expanded by almost 50 percent in the last decade. Not surprisingly, the urbanized area has expanded at an unprecedented rate (Figure 11.2).

Austin has several similarities with AlphaWorld that make it a good candidate for comparison. Although it was originally founded in the 18th century, Austin's population did not grow above one thousand people until the late-19th century. It has undergone rapid growth and urbanization in very recent years, however, as is the case in AlphaWorld. The land area that Austin occupies (625 sq. km) is comparable to that of the sections of AlphaWorld (400 sq. km) for which we have map data. Incidentally, Texas covers 678,051 sq. km of the United States - roughly 1,085 


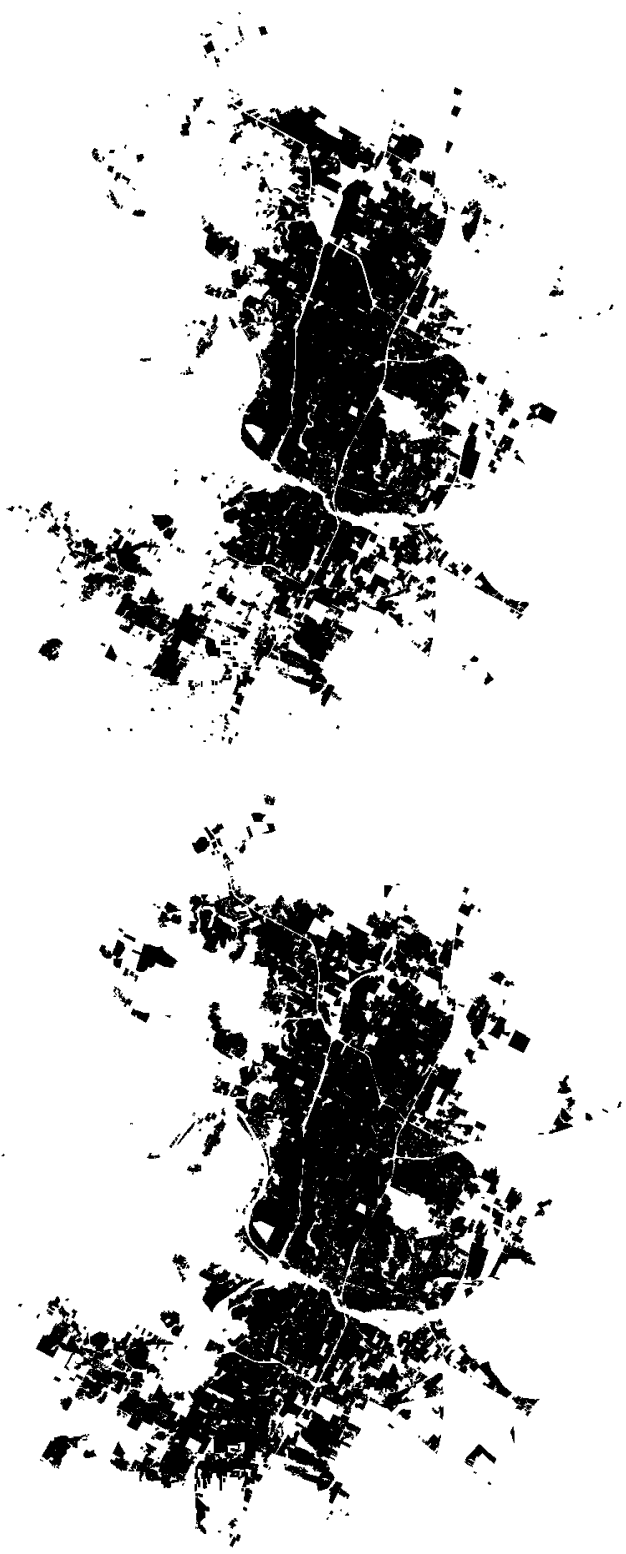

FIGURE 11.2 Urban growth in Austin, Texas, in (a) 1990 and (b) 1995 (growth is shown in gray).

times larger than Austin, which is comparable to the entire area of AlphaWorld, 429,025 sq. $\mathrm{km}$, or 1,073 times the size of its central area.

In addition, Austin shares a flat topographical feature with AlphaWorld, suitable for developing in all directions. Austin is also a poster child for growing concern about sprawl, urban sustainability, and smart growth; these are some of the issues 
that we are interested in exploring in virtual cities. In the following section, we will compare the two cities by extracting and examining their spatial patterns. We will also discuss and compare the change in their growth rate to appreciate the magnitude of their sprawling effect.

\section{ANALYSIS}

\section{Methodology}

While AlphaWorld exists only within the virtual environment of the Internet, as opposed to its counterpart, Austin, Texas, a real city with a tangible urban structure, we can compare their landscape and growth trajectory by using various imageryanalysis techniques and statistical indexes. We focus on the patterns of, as well as the growth dynamics of, the two cities that are represented by the amount and shape of their urbanized area.

At a glance, both AlphaWorld and Austin show a significant increase in their land-use development over a short period of five years. The rapid growth of their urbanized area and the increasingly complex urban structure are also evident in Figures 11.1 and 11.2. The amount of land-use or the urbanized area in AlphaWorld, in particular, has soared between 1996 and 2001.

The fact that we are only considering their urban form could be somewhat restrictive in that the difference in the amount of the actual socioeconomic activities in each land parcel would not be accounted for. Similarly, the shade of urbanized area reflects on the physical extent of the built environment, and not their residents. Measuring its "population" in a virtual city can be a daunting task, as the users can $\log$ in, teleport, and construct buildings wherever they please.

Despite the restriction on the analysis of their socioeconomic and population aspects, interpreting the change in their urban form over time would help maintain an objective perspective for their comparison.

\section{Growth ANalyses}

Using the orthographic images from Figures 11.1 and 11.2, the amount of land-use development was measured for each period of the two cities. Both AlphaWorld and Austin share a significant and recent upsurge in urban development over a short period of five years. Their rapid growth and the increasingly complex urban structure are also evident in Figures 11.1 and 11.2. Between 1996 and 2001, land use in AlphaWorld has soared by 130 percent, and now has a high land-coverage rate of 36 percent (Table 11.2). Austin has also seen a significant increase: 27 percent growth in land-use development between 1990 and 1995 (Table 11.3).

In terms of increases in complexity and land coverage in the two cities, we measured their relative transition by examining the changes in their fractal dimensions (Tables 11.4 and 11.5). Fractal dimension measures the proportion of a space that is filled and the manner in which it is filled; in general, the higher the value, the denser and more complex the structure (Batty and Longley, 1994). Correlation coefficients are an indication of statistical self-similarity — that is, whether a land-cover pattern retains an overall consistency over a wider area, as well as across different scales. 
TABLE 11.2

Land-Use Development in the Central AlphaWorld

\begin{tabular}{lccc} 
Date & $\begin{array}{c}\text { Built Units } \\
(\% \text { of the area: 1048576) }\end{array}$ & $\begin{array}{c}\text { Standard } \\
\text { Deviation }\end{array}$ & $\begin{array}{c}\text { Increase from the } \\
\text { Previous Survey }\end{array}$ \\
mber 1996 & $164138(15.38 \%)$ & 92.66 & \\
ary 1998 & $307502(29.33 \%)$ & 116.09 & $187.34 \%$ \\
t 1999 & $347794(33.17 \%)$ & 120.06 & $113.10 \%$ \\
t 2001 & $376700(35.93 \%)$ & 122.34 & $108.27 \%$ \\
\hline
\end{tabular}

TABLE 11.3

Land-Use Development in Austin, Texas

\begin{tabular}{cccc} 
Year & $\begin{array}{c}\text { Built Area } \\
(\% \text { of the area: 1048576) }\end{array}$ & $\begin{array}{c}\text { Standard } \\
\text { Deviation }\end{array}$ & $\begin{array}{c}\text { Increase from the } \\
\text { Previous Survey }\end{array}$ \\
1990 & $129974(12.40 \%)$ & 84.03 & \\
1995 & $164513(15.70 \%)$ & 92.74 & $126.57 \%$ \\
\hline
\end{tabular}

\section{TABLE 11.4}

\section{Change in Fractal Dimensions of AlphaWorld}

\begin{tabular}{lccc}
\multicolumn{1}{c}{ Date } & Fractal Dimension & Correlation Coefficient & Mean Square Error \\
December 1996 & 1.729353 & 0.998244 & 0.067830 \\
February 1998 & 1.842562 & 0.998675 & 0.062746 \\
August 1999 & 1.865861 & 0.998763 & 0.061389 \\
August 2001 & 1.880098 & 0.998855 & 0.059502 \\
\hline
\end{tabular}

TABLE 11.5

Change in Fractal Dimensions of Austin, Texas

\begin{tabular}{cccc} 
Year & Fractal Dimension & Correlation Coefficient & Mean Square Error \\
1990 & 1.664272 & 0.999663 & 0.028552 \\
1995 & 1.701267 & 0.999608 & 0.031475 \\
\hline
\end{tabular}

The values in Tables 11.4 and 11.5 suggest that both cities have undergone consistent growth and have formed an increasingly complex, yet regular pattern within their respective systems. It should be noted that well-established cities like London had a fractal dimension of 1.737 as early as in 1900 and have only observed a moderate increase since then - its fractal dimension in 1962 was still $\mathrm{D}=1.774$ after 88 years (Abercrombie 1945; Doxiadis 1968). We expect the growth rates of AlphaWorld and Austin to drop dramatically within the next decade, as they are quickly 
approaching the critical limit of space filling: a fractal dimension of $\mathrm{D}=2$, which would mean a complete filling of the entire plane they occupy. It is popularly known that most spatial structures, including urban systems, have an upper threshold for their growth, which is determined by the space occupied by streets, public parks, and other open area (Batty and Longley 1994).

The fractal dimension of AlphaWorld's central mass (Ground Zero and its environs) is already close to 1.9 , which suggests an extremely high density of land use, leaving a small area for streets and open spaces. This is indicative of development patterns to be observed in virtual cities. Because there is no restriction on the form, density, or the structure of their buildings, users of AlphaWorld can afford to build more densely than we would in the real world.

\section{Symmetry ANalysis}

AlphaWorld and Austin also share a common spatial feature in that both of them are highly symmetrical, and this property is evident throughout their growth history. Their similarity ends there, however. The actual shape of land cover in the two cases shows stark contrast, as can be seen in their angular frequency spectra (Figure 11.3). AlphaWorld has a highly diffused spatial structure, with strong regularity around the central axes, whereas Austin has a solid central urban core, surrounded by a less densely inhabited area that sprawls toward every direction in a concentric manner.

Also, if we take the gray level histogram (Table 11.6), AlphaWorld has a higher average value of 91.61 out of a possible 255 (equivalent to 35.93 percent in area coverage) and variance as high as 14,968.05, but its skewness, or an index for the amount of deviation from symmetric histogram distribution:

$$
\mathrm{S}=\left[\sum_{i=0}^{n-1}(i-\mu)^{3} P(i)\right] / \rho^{3}
$$

as well as kurtosis, an index for the degree of concentration of its histogram distribution around the average value:

$$
\mathrm{K}=\left[\sum_{i=0}^{n-1}(i-\mu)^{4} P(i)\right] / \rho^{4}
$$

\section{TABLE 11.6}

\section{Statistical Values of the Gray Level} Histogram

\begin{tabular}{lll}
\multicolumn{1}{c}{ Index } & \multicolumn{1}{c}{ AlphaWorld } & \multicolumn{1}{c}{ Austin, TX } \\
Mean & $\mathbf{9 1 . 6 1 ( 3 5 . 9 3 \% )}$ & $31.61(12.40 \%)$ \\
Variance & $\mathbf{1 4 9 6 8 . 0 5}$ & 7060.97 \\
Skewness & 0.59 & $\mathbf{2 . 2 8}$ \\
Kurtosis & 1.34 & $\mathbf{6 . 2 1}$ \\
\hline
\end{tabular}



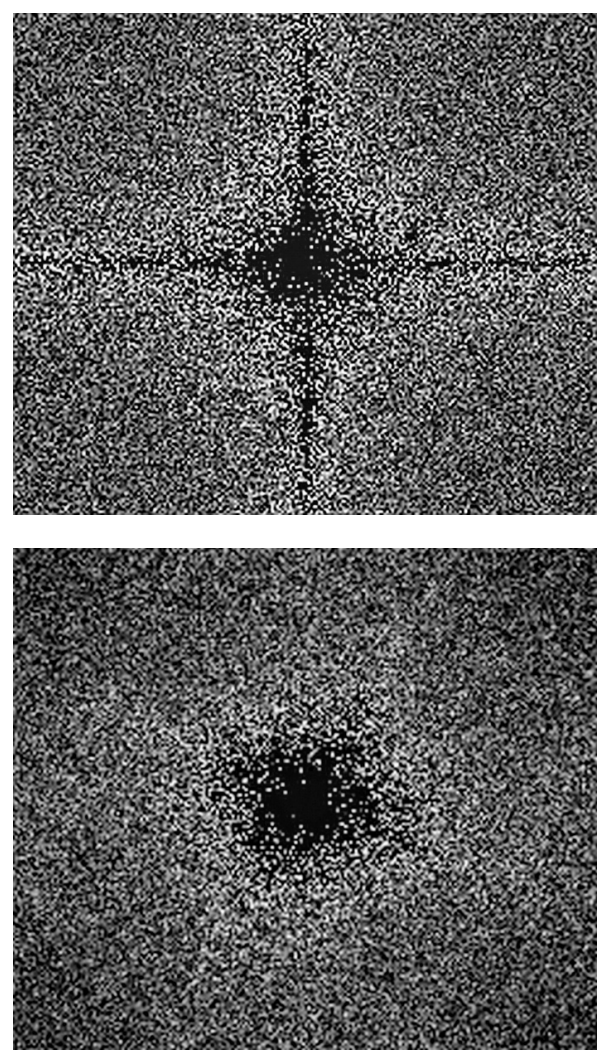

FIGURE 11.3 Visualization of the angular frequency spectrum $(-\pi \sim \pi)$ in (a) the central $400 \mathrm{~km}^{2}$ area of AlphaWorld as of August 2001 and (b) the central area of Austin, Texas, as of 1995 .

are found to be at a lower level of 0.59 and 1.34, respectively. By contrast, the gray level histogram for Austin in 1990 has a lower average value $(E=31.61$ out of a possible 255 , which is equivalent to 12.40 percent in area coverage) and lower variance $(\mathrm{V}=7060.97)$, but much higher skewness $(\mathrm{S}=2.28)$ and kurtosis $(\mathrm{K}=6.21)$. These values confirm the contrast between the urban concentrations found in the two cities, where AlphaWorld has a highly regular structure with much less deviation in its granularity, whereas Austin shows an overall concentric structure but with a varying degree of density.

We also performed vertical and horizontal projections of density distribution of the two cities (Figure 11.4). Density distribution is a widely used notion in molecular physics and biophysics, but its concept is also applicable to other fields such as the measurement of urban structure. In this case, we are measuring the projection of the cumulative density of built environment along the north-south axis and the east-west axis in the two cities. While they both maintain an overall symmetry in their structure, AlphaWorld is much more dispersed over its landscape than Austin, mainly because of the concentration around the outreaching axes and diagonal lines 

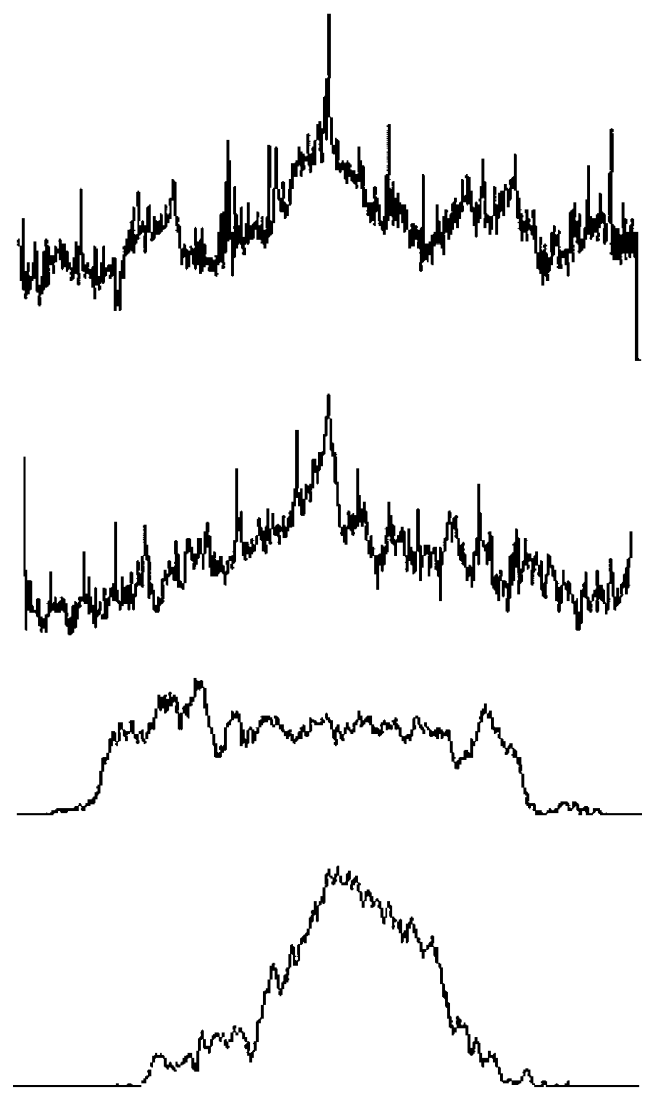

FIGURE 11.4 Projections of density distributions in central AlphaWorld (as of 2001) and Austin (as of 1995): (a) horizontal projection in AlphaWorld, (b) horizontal projection in AlphaWorld, (c) horizontal projection in Austin, and (d) vertical projection in Austin.

(Figures 11.4a, 11.4b); whereas in the case of Austin, there is a significant concentration toward the middle section of the map, that is, its downtown (Figures 11.4c, 11.4d).

On the other hand, Figure 11.4 also shows the relative complexity in the urban landscape of AlphaWorld where its frequency fluctuates at a much shorter interval. This same pattern is evident in the statistical geometrical texture description. The energy level

$$
\left(\sum_{i=0}^{n-1} \sum_{j=0}^{n-1}(i, j)^{2}\right)
$$

across the density distribution is consistent $-0.76(\theta=0, \pi / 4, \pi / 2,3 \pi / 4)$ for Austin (as of 1995); AlphaWorld has a much lower range at $0.40(\theta=0, \pi / 2)$ and 0.36 in diagonal directions, which again confirms the overall diffusion. In addition, the 
inertia levels (i.e., the resistance to changes in density) for AlphaWorld range from $151.47(\theta=0)$ to $221.82(\theta=3 \pi / 4)$, which are a magnitude larger than those of Austin: $16.89(\theta=0)$ to $21.70(\theta=3 \pi / 4)$. All these statistics suggest that, while both AlphaWorld and Austin have highly symmetrical land cover patterns, Austin has a strong concentric structure, as compared to the radial pattern of AlphaWorld.

\section{IMPLICATIONS OF THESE FINDINGS}

\section{LOCATION, LOCATION, LOCATION!}

The results from the two separate analyses suggest that AlphaWorld and Austin have similar growth trajectory yet very different morphological geography. That both cities are booming with rapid increase in their urbanized area gives an impression that their patterns of urbanization are similar in nature.

However, the results from the angular spectrum analyses clearly show that the urban development that is taking place in AlphaWorld is by far the more regular and symmetrical phenomenon than that in Austin. Its users exhibit a strong preference toward locations along the vertical, horizontal, and diagonal axes that radiate from Ground Zero, or the central node. As aforementioned, it reflects the unique address system and preferred way-finding heuristic used in AlphaWorld, where each location is identified by a standard Cartesian coordinate system. As users can teleport to any location with a click of a button, "addresses" with specific combination of coordinate values become a valuable commodity and a new prime location that is desirable in the virtual market. Here in the virtual city, accessibility in the traditional sense of having shorter Euclidian distance gives its way to the significance expressed by a sequence of coordinates.

In contrast, the omni-directional pattern of urban sprawl observed in Austin suggests the persistence of accessibility in the traditional sense that assumes a monotonic distance-decay function of land price originating in the town center. It is indicative of our choice of location in a real city that prefers better access to a strong, growing CBD. At the same time, constant expansion into the suburbs and exurbs implies sprawl on Austin's fringes. The voracious expansion of suburban area is indicative of a phase-shift in preferences for such locations.

That the two cities share a similar growth trajectory but exhibit a different growth pattern shows that both worlds are equally attractive to the residents but that there is a difference in the preferred "location" between the two worlds. After all, patterns of urban growth found in both virtual and real cities reflect a transition in the desired and prime locations that are sought after by their residents.

\section{BUILDING THE IMPOSSIBLE}

The other marked difference between the two cities was found in their vertical profile, or the projections of density distributions (Figure 11.4). For one thing, the frequency range for the density distribution of AlphaWorld is much wider. With the ability to teleport themselves to any location of their choice, users of AlphaWorld have access to geographically farther, remote locations and can, therefore, afford to sprawl outward. This is one luxury we do not have in the real world, as remote 
locations usually mean less accessibility and opportunity. The relatively confined density distribution of Austin shows that the conventional geography is still in tact in this real city.

Besides this, we can also appreciate the difference in the complexity of the wave form of the distribution projections. The dataset from AlphaWorld shows a much denser and vibrant fluctuation in its projection. There are a couple of reasons for this:

1. Many of the buildings designed by the users in a virtual city take an elaborate and intricate form that would be difficult, both technically and financially, to reproduce in the real world.

2. The density and the capacity of each street in a virtual city need not be controlled, as there is no real traffic or need for setback of housing facades to be instituted, and users tend to build much more densely than they would in the real world.

3. In short, buildings in a virtual city tend to adopt different forms and have a larger mass than in the real world. It is likely to remain that way unless a building code or a policy to restrict such building behavior were introduced, although we would not have the necessity to do so in the virtual world.

\section{CONCLUSIONS}

This chapter presented results from a comparison of urbanization trends in two fastgrowing cities, one in real and the other in virtual space: Austin, Texas, and AlphaWorld. Preliminary exploration shows some striking similarities between the two cities. Both are sprawling, at unprecedented rates and in similar styles. The rate of growth is also comparable, as is the area of land cover. Both are developing at lowerthan-average densities on the periphery of the urbanized mass, both have strong central cores amid peripheral expansion, both exhibit symmetrical structures, and both have fractal signatures that indicate a high degree of scattering and fragmentation in land use.

Nevertheless, there are important differences. While both cities are sprawling in a similar fashion, the nature of that sprawl varies considerably between the two cases. Closer analysis reveals significant variation in the pattern of urbanization. AlphaWorld has strong axial orientation, radiating from its central area in horizontal, vertical, and diagonal vertices. Austin, on the other hand, has strong concentric clustering in its spatial distribution. Spectral analysis demonstrates the statistical significance of the variation.

Austin appears to follow the classic stereotype for suburban sprawl in North American cities: a loose and fragmented suburban ring orbiting a central core. AlphaWorld, on the other hand, appears to exhibit two urbanization trends simultaneously: classic sprawl, but organized in a polycentric fashion. The end result, in the case of AlphaWorld is a souplike constellation of fragmented urban clusters.

Of course, we are discussing a virtual city, but the implications for urban planning and policy in the real world could be profound. To a certain degree, the construction of urban space in AlphaWorld is indicative of the types of urban forms that people would like to build in the real world. Our analysis has demonstrated that 
the pattern of urbanization in AlphaWorld is a hybridization of two very popular trends in Western development and settlement patterns. The behavior of people in this cyber city may seem like science fiction: people building without concern for zoning regimes or planning codes, with little care for distance and the capacity to move relatively ubiquitously across the landscape. This is far from far-fetched in real world contexts, however. The situation in AlphaWorld mirrors current trends in the real world: development in the southwest of the United States, for example, appears in many cases to be exercised with little consideration for planning. Decentralized and unplanned growth is the norm, also, on the outskirts of many cities in Latin America. The ubiquity of highways and the public's stated preference for vehicular travel is also creating environments in which space is beginning to matter less and less (Giuliano 1989). To a certain degree, then, AlphaWorld is suggestive of future trends in urbanization and is perhaps the perfect laboratory for exploring what-if questions in urban studies.

\section{REFERENCES}

Abercrombie, P. 1945. Greater London Plan 1944. London: HMSO.

AlphaWorld 3.3. 2006. Activeworlds Corporation, Newburyport, MA, www.activeworlds.com. Batty, M., D. Chapman, S. Evans, M. Haklay, S. Kueppers, N. Shiode, A. Smith, and P. M. Torrens. 2001. Visualizing the city: Communicating urban design to planners and decision-makers. In Planning Support Systems, ed. R. Brail and R. Klosterman, 405-43. Redlands, CA: ESRI Press.

Batty, M. and P. Longley. 1994. Fractal Cities. London: Academic Press.

Batty, M. and N. Shiode. 2003. Population growth dynamics in cities, countries and communication systems. In Advanced Spatial Analysis, ed. P. Longley and M. Batty,, 327-44. Redlands, CA: ESRI Press.

Benenson, I., and P. M. Torrens. 2004. Geosimulation: Automata-Based Modeling of Urban Phenomena. London: John Wiley \& Sons.

Doxiadis, C. A. 1968. Ekistics: An Introduction to the Science of Human Settlements. London: Hutchinson.

Gibson, W. 1984. Neuromancer. New York: Ace Books.

Gibson, W. 1996. Idoru. London: Penguin Books.

Giuliano, G. 1989. New directions for understanding transportation and land use. Environment and Planning A 21:145-59.

Linden Lab. 2007. Second Life (beta), Linden Research, Inc., San Francisco, http://secondlife. $\mathrm{com} /$.

Roelofs, G., and P. van der Meulen. 2002. About the AlphaWorld Map.

Shiode, N. 2000. Urban planning, information technology, and Cyberspace. Journal of Urban Technology 7(2):105-26.

Shiode, N. 2001. 3D urban models: Recent developments in the digital modeling of urban environments in three-dimensions. GeoJournal 52(3):263-69.

Shiode, N. and Yin, L. 2007. In press. Spatial-temporal visualization of built environments. In Understanding Dynamics of Geographic Domains, ed. K. Stewart Hornsby and M. Yuan. Boca Raton: CRC Press.

Stephenson, N. 1993. Snow Crash. New York: Bantam Books.

There (Beta) 2007. There, Menlo Park, CA, www.there.com.

Torrens, P. M. 2002. Cellular automata and multi-agent systems as planning support tools. In Planning Support Systems in Practice, ed. S. Geertman and J. Stillwell, 205-22. London: Springer-Verlag. 
Torrens, P. M., and M. Alberti. 2000. Measuring sprawl. London: University College London, Centre for Advanced Spatial Analysis.

Wrigley, N., T. Holt, D. Steel, and M. Tranmer. 1996. Analysing, modelling, and resolving the ecological fallacy. In Spatial Analysis: Modelling in a GIS Environment, ed. P. A. Longley and M. Batty. Cambridge: GeoInformation International. 